Classification

Physics Abstracts :

17.26

\title{
THEORY OF MOTT TRANSITION : APPLICATIONS TO TRANSITION METAL OXIDES
}

\author{
M. CYROT \\ Institut Laue-Langevin, Cédex 156, 38-Grenoble-Gare, France \\ (Reçu le 22 juin 1971, révisé le 26 août 1971)
}

\begin{abstract}
Résumé. - En utilisant le modèle de Hubbard, nous étudions la transition métal-isolant due aux corrélations entre électrons. Nous ne tenons pas compte des fluctuations de charge mais seulement des fluctuations de spin qui créent sur chaque site un moment magnétique. Cette simplification permet une analogie avec les alliages binaires. A température nulle, nous obtenons successivement un métal non magnétique, un métal antiferromagnétique et un isolant antiferromagnétique lorsque le rapport $U / W$ croît ( $U$ interaction entre électrons, $W$ largeur de la bande). Ceci est dû, en accord avec les idées de Slater, à la partie dépendante du spin du potentiel self consistent. De la même manière qu'il est possible à température nulle d'obtenir une solution antiferromagnétique de plus basse énergie, nous obtenons au-dessus de la température de Néel une solution avec des moments désordonnés sur chaque site. Pour de grande valeur du rapport $U / W$ le système reste isolant au-dessus de la température de Néel. Pour des valeurs intermédiaires de $U / W$, la frontière entre métal et isolant paramagnétique montre que la phase isolante est favorisée à hautes températures à cause de l'entrcpie de désordre. Nous donnons un diagramme de phase schématique pour le modèle de Hubbard et nous discutons l'application de la théorie aux oxydes de métaux de transition.
\end{abstract}

\begin{abstract}
We study the metal-insulator transition due to correlations between electrons using a Hubbard model. Neglecting fluctuations in charge, we only take into account fluctuations in spin density which build up magnetic moments on each site. A close analogy with binary alloys follows from this. At zero temperature, with increasing value of the ratio of the interaction between electrons $U$ to the bandwidth $W$, we obtain successively a non magnetic metal, an antiferromagnetic one and an antiferromagnetic insulator. This is due, as in Slater's idea, to the exchange part of the self consistent potential which cannot have the full periodicity of the lattice. As it is possible to find a solution with lower energy and with antiferromagnetism to Hubbard hamiltonian, one can construct a self consistent solution with random but non zero moments on each site. The alloy analog of the metal insulator transition is band splitting. For large values of the ratio $U / W$, the material remains insulating through the Néel temperature. For intermediate values, the line boundary between a Pauli metal and a paramagnetic insulator shows that the insulating phase is favoured at high temperature because of the entropy disorder. We draw a general schematic phase diagram for the Hubbard model and we discuss the relevance of the theory to transition metal oxides. The main qualitative features are consistent with our theory.
\end{abstract}

Insulator metal transitions are a phenomenon which have been known for quite a while in a wide variety of systems : metal vapour liquid system (mercury) metal ammonia solutions, disordered systems (impurity bands), transition metal chalcogenides. In the last few years there has been considerable interest in transition metal oxides. These oxides form a very interesting class of materials [1]. Their electrical properties range from very good insulator to very good metal. Some of them have an intermediate behaviour and can exhibit a metal-insulator transition with temperature, pressure, or doping.

Besides pratical interest due to possible applications to switches these oxides are of fundamental interest. This stems from the fact they prove to be a striking failure for the elementary Bloch-Wilson theory. It is well known that a metal whose band are either completely full or entirely empty must be an insulator. While it is possible on the basis of the Bloch-Wilson theory to account for metallic behaviour in systems with an even number of electrons per unit cell through band overlap, it is not possible to explain an insulating behaviour when the number of electrons per unit cell is odd. Among the insulating oxides are compounds which on these basis should be metallic. Mott [2] was the first to observe these facts on nickel oxide. For these materials he proposed to abandon Bloch model. The starting point would be a localized ground state wave function or Heitler London one which has the immediate advantage of explaining the insulating behaviour. The reason lies on the fact that if the band is very narrow you do not lose a lot of kinetic energy by localizing the electrons, so an insulating ground state is then favourable. However, as the atoms are brought together the cost in kinetic energy in confining electrons to the atomic sites 
becomes larger until it starts to exceed the repulsion energy gained. At this point the metallic state has lower energy and an insulator metal transition occurs.

A particularly interesting feature of the insulating materials which are not explained in a Bloch-Wilson theory is that they appear to be magnetic. For instance, this is the case of many mono-oxides of transition metal. The insulating behaviour of these materials seems to be strongly connected with magnetism. At this point it seems worth while to define what we shall call a Mott insulator and a Mott transition because a large number of materials exhibit a metal insulator transition although some of them are nonmagnetic in the insulating phase. In general these nonmagnetic materials undergo a lattice phase change which is associated with the insulating behaviour. Although one can argue about the nature of the driving force which induces the lattice phase change, we will not label it a Mott transition. We reserve the word Mott insulator for insulating materials which are not explained by a usual Bloch-Wilson theory and, we believe, are always magnetic. A Mott transition is a transition from a metallic state to a Mott insulator.

Although it is now some twenty years since Mott's original proposal, and there exists a considerable amount of theoretical work, there is, as yet, no satisfactory microscopic theory of the Mott transition. In his original article Mott argued that the transition is due to correlations between electrons. We shall restrict ourselves to this point of view although other mechanisms [1] can be thought of. The occurrence of antiferromagnetism in these insulators is a sign that correlations are probably primarily responsible for the observed facts. So the only articles we will refer to, will discuss this point of view. In this frame, most of the theoretical analysis has started with a model hamiltonian introduced by Hubbard [3]. In this model a localized or Wannier representation is chosen for the electronic wave function. The hamiltonian consists of two terms, one represents the tunelling of electrons from one site to its neighbours and the other the repulsion energy $U$ when two electrons are on the same site.

$$
H=\sum_{i, \sigma} T_{i j} C_{i \sigma}^{+} C_{j \sigma}+U \sum_{i} n_{i \uparrow} n_{i \downarrow}
$$

$C_{i \sigma}^{+}$is the creation operator for an electron of spin $\sigma$ in the atomic state at the $i^{\text {th }}$ lattice site. $T_{i j}$ is the matrix element of the electron transfer between the states at the $i^{\text {th }}$ site and $j^{\text {th }}$ site and $n_{i \sigma}=C_{i \sigma}^{+} C_{i \sigma}$.

In the atomic limit, i. e. for large value of $U / T_{i j}$ and for a single half filled band, there will be an electron localized on each site and the system will be a Mott insulator. In this limit there is an antiferromagnetic coupling energy between the local moments due to Anderson kinetic exchange. Thus at low temperature the system is antiferromagnetic with a Néel temperature $k T_{\mathrm{N}} \sim z T_{i j}^{2} / U$. However, the system is insulating both above and below $T_{\mathrm{N}}$. As $T_{i j} / U$ is increased, the hamiltonian becomes very difficult to solve and there are no exact results. Considerable amount of work has been done and we shall not quote all of them [4]. The main result is due to Hubbard [3] who used a Green function decoupling scheme and completely neglected magnetic ordering. Within his approximation an insulator to metal transition with no discontinuity in the number of free carriers, occurs as $z T_{i j} / U$ goes through a critical value of 1.15. Many improvements of this solution [4] have been tried and many discussions appear. Nonetheless it is not clear that this is the correct result for the Hubbard hamiltonian.

In any case there are some problems linked with Hubbard's solution. First it does not take into account magnetism. Secondly it certainly is incorrect for the metallic phase since it does not properly describe the Fermi surface [5], [6]. Further a temperature induced transition is a rare accident since only change in lattice parameter can drive a phase transition as in Mott's original idea.

Here it is worthwhile to mention Brinkman and Rice's approach [7] to the metal non-metal transition, which used Gutzwiller's variational method [8] of treating the Hubbard Hamiltonian to obtain a non magnetic solution which properly describes the metallic state. The results obtained are to be contrasted with those found by Hubbard: The effective masse becomes large as the correlation increases when Hubbard's approximation leads to a density of states at the Fermi level approaching zero.

In parallel to this line of work following Hubbard there exists another important one following Slater's idea. Slater [9] first pointed out that if a metallic material is antiferromagnetic the effective self consistent Hartree-Fock potential which acts on an electron cannot have the full translational symmetry of the lattice. The exchange part of the potential must have the double periodicity of the sublattice. The first Brillouin zone of the lattice is divided in half and the exchange energy introduces band splitting at the new faces of the reduced subzone. If the exchange energy is large or if the original non magnetic band is narrow this splitting could introduce a real gap in the density of states and thus produce an insulator instead of a metal. We must emphasize that we obtain an insulator only in the ordered magnetic phase. Above the Néel temperature the material becomes metallic. This transition, that we shall call a Slater transition, lies intermediate to a usual Bloch-Wilson one and a Mott one. The insulating behaviour is explained in a Bloch-Wilson theory if one takes into account Hartree-Fock potential. Des Cloizeaux [10] made a particularly detailed study of this transition. Let us emphasize that there is not one Hartree-Fock solution. There exists an infinite number of solutions to the Hartree-Fock equation which are non linear. Des Cloizeaux studied a particular set of Hartree-Fock solutions and determined that at $T=0$ the antiferromagnetic state lies lower than the paramagnetic state. 
Just in the same way that it was possible to find alower energy solution to the Hartree-Fock equations for an antiferromagnet it should be possible to have a self consistent solution with random but non zero moments on each site above the Néel temperature. Depending on the strength of these moments the material could be metallic or insulating. In the following we will show that such a solution is in fact possible. In the metallic phase such moments can build up giving for instance deviation to Pauli susceptibility. For a critical value of these moments the band is not stable and electrons localize on each site. The material undergoes a metal insulator transition. We will develop the theory of this new type of insulator. Essentially it is a magnetic insulator and its properties are connected with magnetic disorder.

Our theory is based on a Hubbard hamiltonian which we believe to contain all the general physical features of the problem. Interactions between electrons can lead to fluctuations in charge density and fluctuations in spin density; we will completely neglect the former and take into account only the latter. So interactions can build up only magnetic moments on each site. Our approach is a self consistent one and develops a close analogy with the theory of alloys. The results are summarized by the general phase diagram of the figure given in part VI. For increasing value of $U / W$ ( $W$ band width), we obtain at zero temperature the succession of three phases: non magnetic metal, antiferromagnetic metal and antiferromagnetic insulator. At finite temperature, for large $U / W$ the phase remains insulating after becoming paramagnetic. The phase boundary, in the temperature versus $U / W$ plane, between the metallic and the paramagnetic insulating phases presents a negative slope and ends at the critical point for decreasing value of the ratio $U / W$.

In part I we develop the general theory and an analogy with alloys. Part II is devoted to the very low temperature region. In part III we study the metallic state and particularly discuss the appearance of magnetism. Part IV is concerned with the insulating phase. The results are summed up in part $\mathrm{V}$ and we draw the general phase diagram that one expects for the Hubbard hamiltonian. The relevance of the theory to transition metal compounds is finally discussed in the last part.

I. General formulation. - As we emphasized above it seems likely that correlations between electrons play the important part in the transition. So we completely neglect the former and use a Hubbard hamiltonian which rests on the following two hypotheses - intracorrelation effects only are effective between electrons sitting at the same time on the same atom - one can define an average effective correlation energy $U$ which gives the average energy difference between pairs of such electrons with parallel or antiparallel spins. It favours parallel spins. These statements recover of course some difficulties arising from the degeneracy of the $d$ band but as a start we will use the simple Hubbard hamiltonian.

Here we will restrict the analyses to thermodynamic properties and calculate the free energy. We want to show that within this band hamiltonian the free energy can have the behaviour of bound electrons: i. e. gives a Heitler-London behaviour. So our main problem is to calculate the partition function defined by

$$
Z=\operatorname{Tr} \mathrm{e}^{-\beta H} .
$$

Let us now set up our approximation. Correlations between electrons can lead to fluctuations in charge and fluctuations in spin. A particularly interesting way [11] to separate both effects is to rewrite the last term of the hamiltonian

$$
U n_{i \uparrow} n_{i \downarrow}=-\frac{U}{4}\left(n_{i \uparrow}-n_{i \downarrow}\right)^{2}+\frac{U}{4}\left(n_{i \uparrow}+n_{i \downarrow}\right)^{2} .
$$

In our model we will completely neglect fluctuations of charges. In the metallic case, this seems reasonable as Coulomb repulsion inhibits fluctuations. In the insulating phase the number of electrons on a site is essentially constant. So we will assume that the last term of equation (3) equals $U / 4$ and we will take into account only fluctuations in spin.

In appendix $\mathrm{A}$ we recall how the partition function can be written in a functional integral form as first pointed out by Hubbard [12]. The main interest of this formulation is to eliminate the two-body potential and to replace it by a time dependent localized field acting on any site $i$. The drawback of this simplification which permit us to treat a simple hamiltonian is that we have to average over all these fields weighted by a gaussian factor.

$$
\begin{gathered}
Z=\int \mathrm{d} \mu_{i s} \exp \left[-\sum_{i s} \frac{\mu_{i s}^{2} U}{4}\right] \operatorname{Tr} T_{s} \exp -\int_{0}^{\beta} H_{s} \mathrm{~d} s \\
H_{s}=\sum_{i j, \sigma, s} T_{i j} C_{i \sigma s}^{+} C_{i \sigma s}+\sum_{i \sigma s}\left(\frac{U}{2}-\mu-\frac{U}{2} \sigma \mu_{i s}\right) n_{i \sigma s}
\end{gathered}
$$

Where $T_{s}$ is the ordering operator for the time " $s$ ». $\frac{U}{2} \mu_{i s}$ is a kind of effective magnetic moment on site $i$. (Cf. appendix B.)

At this point we introduce the second important approximation of this paper. We neglect the "time " dependence of these effective moments, i. e. we are dealing only with Schrieffer's static approximation [13]. This approximation gives the exact result in the two limiting cases, $U=0$ and $T_{i j}=0$ and gives a smooth interpolation between them. We think that the physical results are contained within this approximation which is valid any time fluctuations are unimportant. We believe this is the case in the following except perhaps when we discuss the properties of the metallic phase. 
Now we have to solve essentially the same hamiltonian as Anderson proposed in his study of localization in a random system [14].

The hamiltonian is for one spin direction (with a slight change of zero energy).

$$
H=\sum_{i j} T_{i j} C_{i}^{+} C_{j}+\sum_{i} \frac{U}{2} \mu_{i} n_{i} .
$$

$\mu_{i}$ is a random potential. The only difference is that electrons of spin up and down do not see the same potential but an opposite one.

To calculate the partition function we introduce in the usual manner a coupling constant

$$
\frac{1}{Z} \frac{\partial Z}{\partial \lambda}=\sum_{i \sigma}-\frac{\beta}{2} U \sigma \mu_{i}<n_{i \sigma}>
$$

$\left\langle n_{i \sigma}\right\rangle$ is given by the one electron thermal green function at equal time in the presence of the whole set $\left\{\mu_{i}\right\}$

$$
<n_{i \sigma}>=G_{i i}\left(\tau=0^{-}\right) .
$$

In the absence of the random moments $\mu_{i}$ we have

$$
G_{i j}\left(\omega_{v}\right)=\frac{1}{N} \sum_{k} \frac{\mathrm{e}^{i k_{0}\left(R_{i}-R_{j}\right)}}{i \omega_{v}-\varepsilon_{k}} .
$$

Introducting the explicit notation : $G_{i j}\left(\omega_{v} ; \mu_{1}, \mu_{2}, \ldots, \mu_{n}\right)$ it is straight forward to show that

$$
\begin{gathered}
G_{i j}^{\sigma}\left(\omega_{v} ; \mu_{1}, \ldots, \mu_{i}, \ldots, \mu_{n}\right)=G_{i j}^{\sigma}\left(\omega_{v} ; \mu_{1}, \ldots, \mu_{i}=0, \ldots, \mu_{n}\right)+ \\
\quad+G_{i l}^{\sigma}\left(\omega_{v} ; \mu_{1}, \ldots, \mu_{i}=0, \ldots, \mu_{n}\right) \times \\
\times \frac{-\frac{1}{2} \sigma \mu_{l} U G_{l j}^{\sigma}\left(\omega_{v} ; \mu_{1}, \ldots, \mu_{l}=0, \ldots, \mu_{n}\right)}{1+\frac{1}{2} \sigma \mu_{l} U G_{l l}^{\sigma}\left(\omega_{v} ; \mu_{1}, \ldots, \mu_{l}=0, \ldots, \mu_{n}\right)} .
\end{gathered}
$$

The partition function in presence of the set $\left\{\mu_{i}\right\}$ can be written.

$$
\begin{gathered}
\frac{Z\left(\mu_{1}, \ldots, \mu_{n}\right)}{Z(0, \ldots, 0)}=\prod_{i} Z\left(\mu_{i}\right) \\
Z\left(\mu_{i}\right)=\exp \left\{\sum_{\sigma} \sum_{\omega_{v}} \mathrm{e}^{-i \omega_{v} 0-} \times\right. \\
\left.\times \ln \left[1+\frac{1}{2} \sigma U \mu_{i} G_{i i}^{\sigma}\left(\omega_{v} ; \mu_{1}, \ldots, \mu_{i}=0, \ldots, \mu_{n}\right)\right]\right\} .
\end{gathered}
$$

We transform the sum to an integral in the usual way and obtain the partition function in the form

$$
\begin{gathered}
Z=\int \prod_{i} \mathrm{~d} \mu_{i} \exp -\beta F\left(\left\{\mu_{i}\right\}\right) \\
F\left(\left\{\mu_{i}\right\}\right)=\sum_{i} \frac{\mu_{i}^{2} U}{4}+\sum_{\sigma} \int \frac{\mathrm{d} \omega}{\pi} \frac{1}{1+\mathrm{e}^{\beta \omega}} \times \\
\times \arctan \frac{\frac{U}{2} \mu_{i} \sigma \pi n\left(\omega ; \mu_{1}, \ldots, \mu_{i}=0, \ldots, \mu_{n}\right)}{1+\frac{U}{2} \mu_{i} \sigma P \int \mathrm{d} \omega^{\prime} \frac{n\left(\omega^{\prime} ; \mu, \ldots, \mu_{i}=0, \ldots, \mu_{n}\right)}{\omega^{\prime}-\omega}}
\end{gathered}
$$

$n\left(\omega ; \mu_{1}, \ldots, \mu_{i}=0, \ldots, \mu_{n}\right)$ is the local density of states at point $i$ when $\mu_{i}=0$. It is interesting for the following to introduce the phase shift [15] $\eta_{\sigma}^{i}(\omega)$ due to the localized potential $-\frac{1}{2} U \mu_{i}$ in presence of the other moments $\mu_{j} j \neq i$

$$
\begin{aligned}
F\left(\left\{\mu_{i}\right\}\right) & =\sum_{i} \frac{\mu_{i}^{2} U}{4}+\sum_{\sigma} \frac{1}{2} \int \frac{\mathrm{d} \omega}{1+\mathrm{e}^{\beta \omega}} \cdot \eta_{\sigma}^{i}(\omega) \\
& =\sum_{i} \frac{\mu_{i}^{2} U}{4}+\Delta E_{i}
\end{aligned}
$$

$\Delta E_{i}$ is the change in energy due to the perturbation $-\frac{1}{2} U \mu_{i}$. In general it is impossible to calculate the functional integral. If $F\left(\left\{\mu_{i}\right\}\right)$ is strongly peaked for some value of the set $\left\{\mu_{i}\right\}$ we can approximate the free energy of the system by the free energy for this particular set. This approximation is equivalent to the usual self consistent approximation. This particular set is determined by minimization of $F\left(\left\{\mu_{i}\right\}\right)$ with respect to the effective moment

$$
\frac{\partial F}{\partial \mu_{i}}=\frac{1}{2} \mu_{i} U-\sum_{\sigma} \frac{1}{2} U \sigma<n_{i \sigma}>=0 \quad i=1,2, \ldots
$$

Where we used the fact

$$
\frac{2}{-U \sigma} \frac{\partial}{\partial \mu_{i}} \Delta E_{i}=\left\langle n_{i \sigma}\right\rangle
$$

i. e.

$$
\mu_{i}=\left\langle n_{i \uparrow}>-<n_{i \downarrow}>\quad i=1,2, \ldots\right.
$$

These equations 16 are the self consistent equations of our problem. Of course $\left\langle n_{i \sigma}\right\rangle$ is a function of the whole set $\left\{\mu_{i}\right\}$ but in principle this set of equations determines $\mu_{i} i=1,2, \ldots$ The procedure we used is the usual one which gives a self consistent approximation. Equation 16 could have been deduced at once from equations 1 and 3 using a Hartree-Fock factorization.

We preferred to use a relatively more complicated formalism which has the advantage of leading directly to the thermodynamical quantities that a study of phase transitions require. In particular the entropy term comes out of the theory contrary to a simple Hartree-Fock's one.

From equation 12 we see that $F$ is an even function of all $\mu_{i}$. So if we have a particular set which minimizes $F$ all sets where we change the sign of one moment minimize $F$. When all the sites can be considered as equivalent $\mu_{i}$ is independent of site $i$ and we have $\mu_{i}= \pm \mu$. As a result our problem reduces to an alloy one. The only difference is that $\mu$ must be determined self consistently but we can rest very heavily on known results for alloys [16].

Many general conclusions can be drawn at this point. At low temperature when $\mu$ is different from zero there is magnetic order. This happens as we will show for not too small value of $U / W$ ( $W$ band width). For one electron per atom the phase is antiferromagnetic and our problem reduced to an ordered alloy 
one. Our results are essentially similar to Slater's ones [9]. When $U \mu$ is large enough the band splits and we obtain an insulator. This will be discussed in Part III.

The most interesting possibility of this model lies on the fact that we can also describe a magnetically disordered insulator. In our analogy a split band limit can occur in a disordered alloy. This rests on the well known localization theorem [16] for alloys. For a binary alloy of the type considered, the energy levels lie within the energy bands of the pure metals. For the disordered alloy the density of states is zero in no interval within these bands. In our case the split band limit is equivalent to an insulator. We will show that for large ratio $U / W$ the entropy disorder favours large $U \mu$ which leads to band splitting. So whatever the temperature, i. e. in the ordered or disordered state we obtain an insulator for large $U / W$.

If we associate band splitting and appearance of an insulating phase our criterion for the transition to a disordered insulating phase is $\mu U=W$. However if for the ordered phase this assimilation is certainly true, for the disordered phase the possible existence of localized states within the band makes this criterium an upper limit. We will discuss this possibility in the theory of the insulating phase (part V).

II. Very low temperature region. - At zero temperature many simplifications occur. One can always assume perfect ordering if magnetism exists and the hamiltonian becomes periodic. Thus the interest of a detailed study of this case stems from the exact calculations that one can perform. Some studies have been done [10]-[17] under assumptions which are equivalent to ours at zero temperature. So our results are formally similar to those of these authors. However we give there another range of validity. The hamiltonian for one spin direction is :

$$
H=\sum_{k} \varepsilon_{k} C_{k}^{+} C_{k}+\sum_{i} \frac{1}{2} U \mu \mathrm{e}^{i p \cdot R_{i}} n_{i}
$$

$\mu$ must be determined by the self consistent equation 16 . In equation 17 we have assumed an antiferromagnetic order and $p$ is half a reciprocal lattice vector. Except in special case we obtain two critical values for the ratio $U / W$. Below the first one no magnetism occurs, i. e. the self consistent solution of equation 16 is $\mu=0$. For intermediate value of this ratio, one obtains an antiferromagnetic metal and for large value an antiferromagnetic insulator because a gap opens up in the density of states [16].

Hamiltonian [17] is easily diagonalized using a pairing of states $k$ and $k+p$

$$
\gamma_{k}=u_{k} C_{k}+v_{k} C_{k+p}
$$

and we obtain the eigen energy

$\lambda_{k}=\frac{\varepsilon_{k}+\varepsilon_{k+p}}{2} \pm \frac{\operatorname{sg}\left(\varepsilon_{k}-\varepsilon_{k+p}\right)}{2} \sqrt{\left(\varepsilon_{k}-\varepsilon_{k+p}\right)^{2}+U^{2} \mu^{2}}$ the self consistent equation which determines $\mu$ becomes

$$
1=\frac{2}{N} \sum_{[k]} \frac{U\left[n\left(\lambda_{k}^{-}\right)-n\left(\lambda_{k}^{+}\right)\right]}{\sqrt{\left(\varepsilon_{k}-\varepsilon_{k+p}\right)^{2}+U^{2} \mu^{2}}}
$$

$n$ is the Fermi function at zero temperature.

The sum is performed on the magnetic Brillouin zone i. e. a reduced zone. Equations 18 and 19 are similar to those of reference [17]. In reference [17] and [8] the energy associated with this solution has been calculated in one dimension to compare it with the exact solution of Lieb and $\mathrm{Wu}$ [19] on the Hubbard hamiltonian in this case. One finds a reasonable agreement and this result justifies somewhat our assumption of neglecting density fluctuations.

Let us remark that one has to be careful when using a simple tight binding approximation for $\varepsilon_{k}$. For a band in a simple cubic or body centered cubic one has :

$$
\varepsilon_{k}+\varepsilon_{k+p}=0 .
$$

Equation 19 shows that the insulating behaviour appears with magnetism. As the right hand side of equation 20 diverges for $\mu=0$, we find that the ground state is antiferromagnetic and insulating even for $U$ infinitesimally small. In general a finite value of $U$ is required to obtain antiferromagnetism. $U$ must be equal to $2 \mu_{B}^{2} / \chi_{p}$ where $\chi_{p}$ is the susceptibility for wave vector $p$. When the ratio $U / W$ is larger than a second critical value a gap opens up in the density of states. For F. C. C. crystal with ferromagnetic III plane stacked antiferromagnetically this happens for

$$
U \mu>0.66 \mathrm{~W} \text {. }
$$

We also notice that this description permits in a band model localized magnetic moments with non integral spin even in the insulating phase. From equation 20 one can verify that $\mu$ is equal to the atomic magnetic moment only in the limit

$$
U / W \rightarrow \infty \text {. }
$$

Formula 20 can be easily generalized at finite temperature. For a simple cubjc we have :

$$
1=\frac{U}{N} \sum_{[k]} \frac{\operatorname{th} \beta / 4 \sqrt{\left(\varepsilon_{k}-\varepsilon_{k+p}\right)^{2}+U^{2} \mu^{2}}}{\sqrt{\left(\varepsilon_{k}-\varepsilon_{k+p}\right)^{2}+U^{2} \mu^{2}}} .
$$

But we must emphasize that we have assumed at the beginning an antiferromagnetic order. So this equation is only valid at low temperature when the long range order is not destroyed i. e. for temperature smaller than the Néel temperature. Even in this case equation 21 is approximate because some electrons are excited and we obtain disorder. It is not possible, within this simple calculation, to deduce from equation 21 a Néel temperature or a temperature for a metal insulator transition. The coupling between moments must be calculated in order to obtain the 
Néel temperature. We will do this, both in the metallic and insulating phase, and will compare the free energy of the different phase. It is our main difference with the quoted reference. In particular we will not find any metal insulator transition for large value of $U / W$; the system is always insulating in an antiferromagnetic or paramagnetic phase.

III. Theory of metallic phase. - In this part we want to investigate the magnetic properties of the metallic phase. This is particularly important for drawing the general phase diagram and calculating the Néel temperature. We have just shown that the metallic phase can be magnetic or non magnetic at low temperature. For low value of $U / W$ the phase is non magnetic. This remains true at all temperature and the phase has the usual Pauli susceptibility. When the low temperature phase is magnetic and metallic there are two possibilities for the apperarance of magnetism with decreasing temperature. In the first one the non magnetic phase becomes antiferromagnetic below a temperature $T_{\mathrm{N}}$. In the second one localized moments appear in the non magnetic phase. These moments interact through a Ruderman-Kittel interaction which make them ordered at a critical temperature $T_{\mathrm{N}}$. We calculate that these localized moments are stable only below a temperature $T_{\mathrm{L}}$ $\left(T_{\mathrm{L}}>T_{\mathrm{N}}\right)$

We get successively these two different behaviours for increasing value of $U / W$. They correspond to a first and to a second order transition. We have already applied this theory [21] to discuss transition metal and to emphasize that a Heisenberg behaviour can stem from a band model much of the discussion is still valid.

To illustrate these different behaviours let us go back to equation 12 and assume for the moment that we can neglect all term which connect the local field on different sites that is :

$$
\begin{aligned}
F\left(\left\{\mu_{i}\right\}\right) & =\sum_{i} F_{1}\left(\mu_{i}\right)=\sum_{i} \frac{\mu_{i}^{2} U}{4}+\sum_{\sigma} \int \frac{\mathrm{d} \omega}{\pi} \frac{1}{1+\mathrm{e}^{\beta \omega}} \times \\
& \times \arctan \frac{\frac{1}{2} \pi \sigma \mu_{i} U n(\omega)}{1+\sigma \mu_{i} \frac{U}{2} P \int \mathrm{d} \omega^{\prime} \frac{n\left(\omega^{\prime}\right)}{\omega^{\prime}-\omega}}
\end{aligned}
$$

$n(\omega)$ is the density of state of hamiltonian (6) when the whole set $\left\{\mu_{i}\right\}$ is equal to zero. We evaluate the functional integral (11) by means of the saddle point method if there is a deep minimum for the function $F_{1}$. This function is even and for large value of $T$, $F_{1}\left(\mu_{i}\right)$ has a single minimum at the origin so that state $\mu_{i}=0$ is most heavily weighted. For $T$ smaller than $T_{\mathrm{L}}$ there rise two symmetrical minimum $\pm \mu_{0}$ corresponding to spin up and spin down. The susceptibility corresponding to these two regimes gives from a Pauli law to a Curie law with a temperature dependant moment [13]. The temperature $T_{\mathrm{L}}$ is given by
$1+U \int \mathrm{d} \omega \int \mathrm{d} \omega^{\prime} \frac{f(\omega)-f\left(\omega^{\prime}\right)}{\omega-\omega^{\prime}} n(\omega) n\left(\omega^{\prime}\right)=0$.

One can see that $T_{\mathrm{L}}$ is zero for $U / W$ smaller than a critical value which depends on the shape of the band. We recall that this formula is only valid in the metallic state i. e. for not too large value of the ratio $U / W$.

Within the static approximation the validity of this one site approximation can be discussed by considering coupling between localized moments. The next approximation to equation 10 is [13] :

$$
\ln \left[1+\mu_{i} \sigma \frac{U}{2} G_{i i}^{0 \sigma}-\frac{1}{4} \mu_{i} \mu_{j} U^{2} \frac{G_{i j}^{0 \sigma} G_{j i}^{0 \sigma}}{1+\mu_{j} \frac{U}{2} \sigma G_{j j}^{0 \sigma}}\right]
$$

In fact it is easy to show that one can write :

$$
\begin{aligned}
Z=\int \prod_{i} \mathrm{~d} \mu_{i} \exp -\beta & \left\{\sum_{i} F_{1}\left(\mu_{i}\right)+\sum_{i j} F_{2}\left(\mu_{i}, \mu_{j}\right)+\right. \\
& \left.+\sum_{i j k} F_{3}\left(\mu_{i}, \mu_{j}, \mu_{k}\right)+\cdots\right\}
\end{aligned}
$$

where the ratio of two successive terms is small. When $F_{1}\left(\mu_{i}\right)$ hast wo symmetric minimum $\pm \mu_{0}$ we can approximate $\mu_{i}$ and $\mu_{j}$, in the smaller quantity $F_{2}$ by $\mu_{0} \sigma_{i}$ and $\mu_{0} \sigma_{j}$ with $\sigma_{i}= \pm 1, \sigma_{j}= \pm 1$ and neglect all interactions of order greater than two. One can write

$$
F_{2}=-\sum_{i j} J_{i j} \sigma_{i} \sigma_{j}
$$

The Fourier transform of $J_{i j}$ is given by

$$
J(q)=\frac{1}{2} U^{2} \mu^{2} \frac{1}{N} \sum_{k} \frac{f\left(\varepsilon_{k}\right)-f\left(\varepsilon_{k+q}\right)}{\varepsilon_{k}-\varepsilon_{k+q}} .
$$

We obtain a Ruderman-Kittel like interaction between localized moments. Thank to it we will get a transition to a magnetic ordered state in decreasing temperature.

When the ratio $U / W$ is not large enough it is unfavorable to create localized moments in a magnetically disordered state. However it can be worthwhile to create ordered localized moments because of the energy gained by magnetic ordering. That is $T_{\mathrm{L}}$ equal the transition temperature to the ordered magnetic phase $T_{\mathrm{N}}$.

To show this possibility we always neglect interaction of order greater than two and we restrict, in the calculation of the partition function, to value $\mu_{k}=\mu \sigma_{k}$, $\sigma_{k}= \pm 1$. Then we look at minimum of $F_{1}+F_{2}$ as a function of $\mu$. The sum over $\sigma_{k}$ leads to the free energy of an Ising model $\mathcal{F}(\mu)$ with interaction given by equation 27 .

$$
Z=\int \mathrm{d} \mu \exp -N\left\{F_{1}(\mu)+\mathcal{F}(\mu)\right\} .
$$

This integral can be done by a saddle point method. One obtain a non zero value of $\mu$ if the energy needed to create a localized moment is less than the energy 
gained by magnetic ordering. In creating directly ordered moments we obtain magnetism for a lower value of $U / W$ than that given by equation (23).

For the special case of a parabolic density of states

$$
\begin{aligned}
n(\omega) & =\frac{8}{\pi w^{2}} \sqrt{\frac{w^{2}}{4}-\omega^{2}} & & |\omega|<\frac{w}{2} \\
& =0 & & |\omega|>\frac{w}{2}
\end{aligned}
$$

we can apply our results. Equation 23 gives for the existence of localized moments in a disordered state a minimum ratio of $U / W$ of $3 \pi / 16=0.59$. Taking into account the energy gained by magnetic ordering this ratio becomes of the order of 0.24 . So we obtain the qualitative diagram of figure 1.

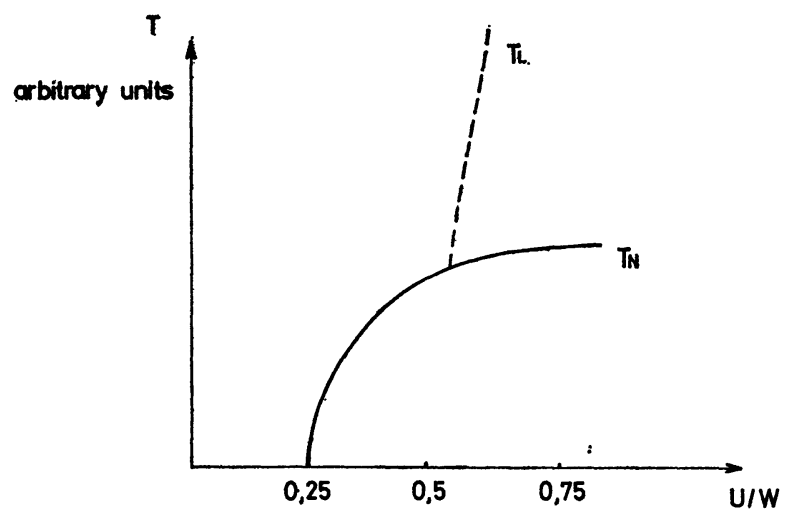

FIG. 1. - Schematic phase diagram for the metallic phase. The dashed curve does not represent a phase transition but the temperature below which localized moments are stable.

IV. The insulating phase. - In the usual theory an insulator is a material whose band are either completely full or entirely empty. Mott was the first to point out that another type of insulator can exist. It is typified by an array of hydrogen atoms in the atomic limit. Electrons are localized on each site although in a Bloch theory one obtains a half filled band. So this type of insulator is only due to correlation and is magnetic.

In the ordered phase agreement between these two types occurs because the superimposed exchange field due to antiferromagnetism splits the first Brillouin zone. Slater's description with Bloch wave functions is equivalent to Mott's with a Heitler-London ground state. Our results of part II illustrate this point. Slater's idea seems simpler to deal with and to explain non integral spin in the insulating phase but is misleading in the sense that it suggest incorrectly that the crystal will show metallic conductivity above the Néel temperature.

Mott description has the advantage of permitting a paramagnetic insulator but is very difficult to set up mathematically. We will show that our formalism, which developed an analogy with an alloy and which gave physically the same result as Slater's idea in the ordered phase, can be extended to deal with the paramagnetic case. The formulation is different from Mott's but it lies on the same physic and permits effective calculation.

In part I we developed an analogy with alloys because the magnetic moment on each site acts as a local potential. So we can applied known results on this problem. As we quoted above the localization theorem for binary alloy gives an exact criterium for a split band which is equivalent to insulating behaviour. We obtain the split band if

$$
\mu U>W
$$

This important inequality means that we get insulating behaviour for a given moment when $U$ is large enough. As $\mu$ has a maximum value which is given by Hund's rule, there is a minimum value of $U$ below which no insulating phase can appear. This remark will be used in the following.

Up to now we have associated band splitting and appearance of an insulating phase. This is certainly true for the ordered phase but probably wrong in the disordered one. This stems from the fact that it is now generally believed that a continuous density of localized states can exist in a disordered system. If the Fermi level lies within these states it does not define a metal but rather an insulator. In our case the localization theorem does not say anything about the nature of the states localized or extended throughout the lattice. Before band splitting, the states at the minimum of the density of states which occurs in the middle of the band may be localized. So we would get an insulating behaviour with a Fermi level located in the middle of the band. Anderson [22] was the first to give strong arguments in favour of a continuous density of localized states. Later Mott [23] introduced the concept of a critical energy separation in a band localized from non localized states. So in our magnetically disordered case, band splitting is not the exact criterion for the metal insulator transition. One must rather know the nature of the states at the Fermi level.

Anderson [14] gave a definition of the concept of localization : if one put an electron on a particular site at time zero, it has a finite probability to be on the same site at infinite time. To study this problem he emphazised [22] that it is wrong to study averages but instead all quantities must be characterised by their probability distribution functions. We have seen in part II that a natural quantity one has to introduce is the local density of states on site $i$ without the perturbation on this site. This is easily seen from equation 12 .

If the self consistent magnetic moment $\mu_{i}$ is large enough to create a bound state of energy $E_{i}^{B}$ out of the local density of states $n\left(\omega ; \mu_{1}, \ldots, \mu_{i}=0, \ldots, \mu_{n}\right)$ the free energy has an extra contribution

$$
T \ln \left[1+\mathrm{e}^{-\beta E_{i}^{B}}\right]
$$


this state corresponds to a localized one in Anderson's sense because the phase shift has a discontinuity of $\pi$. So the free energy shows two contributions one due to bounds electrons and the other to itinerant ones.

This criterium must replace equation 29. Unhappily it is not an explicit one because it lies on the unknown quantity, the local density of states which one has to approximate. However our derivation has the immediate advantage to prove Mott assumption about the critical energy separating localized from non localized states.

The general theory we described would tend to divide the insulators in two differents types: The first one described an insulator due to band splitting where the concept of filled or empty bands exist. The second type is analogous to amorphous semiconductors [23] : there is no gap in the density of states but it exists a « mobility gap ».

The Aтомic Limit. - We will now do some explicit calculations and derive some known results in this limit.

To calculate a possible bound state on site $i$ we have to solve the equation

$$
\frac{2}{\sigma U \mu_{i}}=P \int \mathrm{d} \omega^{\prime} \frac{n\left(\omega^{\prime} ; \mu_{1}, \ldots, \mu_{i}=0, \ldots, \mu_{n}\right)}{\omega^{\prime}-\omega}
$$

where $n$ is the local density of states if $\omega$ is out of the band we have the well known expansion :

$\int \mathrm{d} \omega^{\prime} \frac{n\left(\omega^{\prime}\right)}{\omega^{\prime}-\omega}=-\left(\frac{M_{0}}{\omega}+\frac{M_{1}}{\omega^{2}}+\frac{M_{2}}{\omega^{3}}+\frac{M_{3}}{\omega^{4}}+\cdots\right)$

$M_{p}$ is the $p^{\text {th }}$ moment of the local density of states and is defined by

$$
M_{p}=\left\langle i\left|H^{p}\right| i\right\rangle .
$$

$H$ is the hamiltonian with $\mu_{i}=0$ and $|i\rangle$ is the Wannier function on site $i$. The first few moments are easily calculated if one take into account only non zero value of $T_{i j}$ for nearest neighbours.

$$
\begin{gathered}
M_{0}=1, M_{1}=0, M_{2}=\sum_{j} T_{i j}^{2}, \\
M_{3}=\sum_{j} \frac{1}{2} \mu_{j} U T_{i j}^{2} .
\end{gathered}
$$

In the atomic limit $T_{i j}=0$ one gets

$$
\omega=-\frac{U \mu_{i}}{2}
$$

i. e. the atomic level. For spin down electrons the hamiltonian is identical. The level is the same and there is a complete degeneracy between spin up and down. Let us now investigate the small $T_{i j}$ value. If we restrict to second moment we just obtain a shift of the atomic level for both spins up and spins down

$$
\omega=-\frac{U \mu_{i}}{2}-\frac{2 M_{2}}{U \mu_{i}} .
$$

If we take into account the third moment, spins up and spins down become inequivalent and we obtain difference between both level given by

$$
\Delta \omega=4 \sum_{j} \mu_{j} \frac{T_{i j}^{2}}{U \mu_{i}} .
$$

The degeneracy is raised at low temperature and the material is antiferromagnetic with a Néel temperature of the order of $T_{i j}^{2} / U$. Both results are well known but this show how one can obtain an insulating phase and what is the magnetism of this phase. The phase is still insulating above the Néel temperature. The coupling between moments could have been also deduced from equation 23 with as zero order the atomic green function and calculating $G_{i j}$ to second order in $T_{i j}$.

In the general case the local density of states is made up of extended states surrounded by localized states which represent the contribution of the localized electrons on site near by the particular site we consider. In a first approximation valid for small value of $T_{i j}$ we can neglect the contribution of the localized states and take into account only extended ones. The reason is that the contribution on site $i$ of a localized state on site $j$ is proportional to $G_{i j}^{2}$. In this limit this is a small quantity. Roughly speaking the width of the band of extended states is $\left(\sum_{j} T_{i j}^{2}\right)^{1 / 2}$ and to extract a bound state from this band we get the same kind of criterium as equation 28 but the band width is smaller because the tail is made up of localized states which do not contribute to the local density of states.

It is interesting to note that in this description the gap measured by optical absorption does not equal the gap measured by conductivity due to the possible existence of localized states. This property is due to disorder and an analogy can be drawn between this insulating phase and amorphous material. The only difference is that in the latter case the $\mu$ 's are properties of the system and in the former it has to be determined self consistently. However it seems likely that some of the predictions about amorphous systems could be extended to explain properties of magnetic insulators in the disordered phase.

V. Schematic phase diagram. - We want now to sum up our results and to draw a schematic phase diagram for the Hubbard model. At zero temperature we obtain with increasing value of the ratio $U / W$ a metallic Pauli region up to $U / W=0.2$ then a magnetic metallic one which is antiferromagnetic for a half filled band. At a critical value of 0.6 the material becomes insulating. We will investigate in the following the influence of temperature. We already discuss the disappearance of the magnetic ordered state both in the metallic and insulating region. So we will now calculate the phase boundary between the paramagnetic 
insulator and the Pauli metal. Our main result is that the high temperature phase is insulating due to the large entropy disorder of this phase [25]. This boundary terminates at a critical point for the low value of $U / W$ because of the inequalities that we derive above.

For this purpose we have to compare the free energy of the metallic phase and of the insulator. The first one is [24]

$$
F_{M}=-2 T \int \mathrm{d} \omega n(\omega) \ln \left[1+\mathrm{e}^{-\beta \omega}\right]
$$

and the second one

$$
\begin{aligned}
F_{I}=\sum_{i} \frac{U \mu_{i}^{2}}{4}-N T \ln 2+ \\
\quad+T \sum_{i} \int \mathrm{d} \omega n_{i}(\omega) \ln \left[1+\mathrm{e}^{-\beta \omega}\right]- \\
\quad-T \sum_{i} \ln \left[1+\mathrm{e}^{-\beta E_{B}^{i}}\right]-\ln \left[1+\mathrm{e}^{+\beta E_{B}}\right] .
\end{aligned}
$$

Due to the entropy disorder $\ln 2$ the insulator is more stable at high temperature for relatively low value of $U$ we get

$$
T_{M} \sim \frac{W^{2}}{U \mu} .
$$

The temperature of the metal non metal transition increases with decreasing $U$. As we know that we cannot obtain an insulating phase if $U<W$, we get a critical point where the first order phase transition ends [26]. On the other limit for large value of the ratio $U / W$ this line terminates at the intersection with the phase boundary of the antiferromagnetic insulator. So we can draw the schematic diagram of figure 2 .

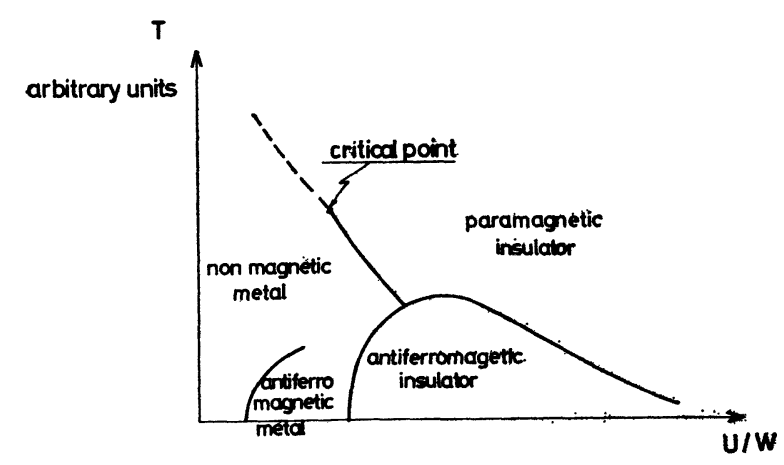

FIG. 2. - General schematic phase diagram.

VI. Possible applications to transition metal oxides. - Our theory describes the effect of correlation in narrow bands and transition from a band metal to a localized magnetic insulator. Transition metal oxides are good candidates for the relevance of the theory to experiments. The $d$ functions form a relatively narrow $d$ band which is responsible for both the electrical and the magnetic properties of the material [1]. $\mathrm{V}_{2} \mathrm{O}_{3}$ is a typical case which presents this transition and has been particularly studied [25], [27]. The general phase diagram of reference 20 for $\left(\mathrm{V}_{x} \mathrm{Cr}_{1-x}\right)_{2} \mathrm{O}_{3}$ has qualitative features of our figure 2 . The main difference with the predicted schematic phase diagram is the absence of the antiferromagnetic metallic phase. This can be viewed as an effect of the lattice phase change which occurs at the metal-antiferromagnetic insulator in this compound. With the transition is associated a sharp volume increase [25], [27]. There is a discontinuity in the $U / W$ ratio which is illustrated in figure 36 of reference [28], by an invisible region where this antiferromagnetic metallic phase should have occurred. A detailed discussion of the lattice instability linked with the Mott transition will be discussed in a forthcoming paper.

Appendix A. - As a start we have Hubbard Hamiltonian (eq. 1) that we rewrite :

$H=\sum_{i j \sigma} T_{i j} C_{i \sigma}^{+} C_{j \sigma}+\frac{U}{4}\left(n_{i \uparrow}+n_{i \downarrow}\right)^{2}-\frac{U}{4}\left(n_{i \uparrow}-n_{i \downarrow}\right)^{2}$.

We want to calculate the partition function

$$
Z=\operatorname{Tr} \mathrm{e}^{-\beta H} \text {. }
$$

Stratonovich [29] then Hubbard [12] developed a functional integral method for calculating $Z$ which uses the identity valid for any bounded operator $a$.

$$
\mathrm{e}^{\alpha a^{2}}=\sqrt{\frac{\alpha}{\pi}} \int_{-\infty}^{+\infty} \mathrm{d} x \mathrm{e}^{-\alpha x^{2}+2 \alpha x a} .
$$

We want to apply it to the last term of the hamiltonian. As this term does not commute with the first two, we first use the Feynman time ordering trick.

$$
\mathrm{e}^{A+B}=T_{s} \exp \int_{0}^{1} \mathrm{~d} s\left(A_{s}+B_{s}\right) .
$$

Where $s$ is a fictious time, $T_{s}$ is a chronological ordering which order products. The $A_{s}$ and $B_{s}$ can be treated as commuting operators so long as they are acted on by the $T_{s}$ operator in the end. Using this trick and the identity just above we obtain equation 5 and 6 . Here we have neglected fluctuations in charge : i. e. we replaced the second term of the hamiltonian by $U / 4$. We believe that self consistence and links with other works are better taken into account through Hamman [11] way of line - arizing (using equation 3) than through Schrieffer approach [13].

Appendix B. - We want to calculate the magnetic susceptibility which is given by

$$
\left.\chi=\frac{1}{\beta} \frac{\partial^{2} \ln Z}{\partial h^{2}}\right]_{h=0} .
$$

One can easily see from equation 5 that the applied field $h$ enters $Z$ additively with the local moment. One has to do the replacement

$$
\mu_{i} \rightarrow \mu_{i}+\frac{2 \mu_{B} h}{U}
$$


Therefore we have

$$
\frac{Z^{h}\left(\left\{\mu_{i}\right\}\right)}{Z_{0}^{h}}=Z^{h=0}\left(\left\{\mu_{i}+\frac{2 \mu_{B} h}{U}\right\}\right) / Z_{0}^{h}
$$

the only $h$ dependence is displayed explicitely in the Gaussian weight factor. In the limit where one can neglect interactions between moments we get the simple result

$$
\chi=\chi_{0}+\sum_{i} \frac{\mu_{B}^{2} \mu_{i}^{2}}{T}-\frac{\mu_{B}^{2} ! !}{U}
$$

The brackets represent a thermal average.

In the metallic phase $\chi_{0}$ is the band susceptibility. This result shows that $\mu_{i}$ is a kind of effective moment on site $i$, which if $\mu_{i}$ is temperature independant give rise to a Curie law.

\section{References}

[1] AdLer (D.), Solid State Physics, 1968, 21, 1.

[2] Mott (N. F.), Proc. Phys. Soc. (London), 1949, A 62, 416; Can. Journal of Physics, 1956, 34, 1356 ; Phil. Mag., 1961, 6, 287 ; Rev. Mod. Phys., 1968, 40, 677.

[3] Hubbard (J.), Proc. Roy. Soc., 1963, A 276, 238 ; ibid., 1964, A 277, 237 ; ibid., 1964, A 281, 401.

[4] Doniach (S.), Adv. in Physics, 1969, 18, 819.

[5] Herring (C.), in ( Magnetism ) vol. 4, edited by G. T. Rado and H. Suhl (Academic Press. New York, 1966).

[6] Edwards (D. M.) and Hewson (A. C.), Rev. Mod. Phys., 1968, 40, 810.

[7] Brinkman (W. F.) and Rice (T. N.), Phys. Rev., 1970, B 2, 4302 .

[8] GutzWiller (M. C.), Phys. Rev., 1965, 137, A 1726.

[9] Slater (J. C.), Phys. Rev., 1951, 82, 538.

[10] Des Cloizeaux (J.), J. Physique, 1959, 6, 606; ibid., $1959,8,751$.

[11] Hamman (D. R.), Phys. Rev., 1970, 2, 1373.

[12] Hubbard (J.), Phys. Rev. Letters, 1959, 3, 77.

[13] SChriefFer (J. R.), Unpublished lecture notes C. A. P. Summer School Banff 1970.

WANG (S. Q.), Evenson (W. E.), SchriefFer (J. R.), Phys. Rev. Letters, 1969, 23, 92 ; J. Appl. Phys., 1970, 41, 1199.

[14] Anderson (P. W.), Phys. Rev., 1958, 109, 1492.

[15] Friedel (J.), Rendiconti della Scuola Internazionale di Fisica E. Fermi 37, Corso (1966).

[16] Soven (P.), Phys. Rev., 1967, 156, 809; ibid, 1969., 178, 1136.

Velicky (B.), KikPatrick (S.) and Ehrenreich (H.), Phys. Rev., 1968, 175, 747.

[17] Langer (W.), Plischke (M.) and Mattis (D.), Phys. Rev. Letters, 1969, 23, 1448.

[18] Johansson (B.) and BergGren (K. F.), Phys. Rev., 1969, 181, 855.

[19] Lieb (E.) and Wu (F.), Phys. Rev. Letters, 1968, $20,1445$.

[20] Herring (C.), in Magnetism, Vol. IV, edited by G. T. Rado and H. Suhl (Academic Press, New York, 1966).

[21] Cyrot (M.), Phys. Rev. Letters, 1970, 25, 871 ; International Conference on low temperature physics $L T 12$ (1970) to be published. In our first reference PRL it was not clear that our theory was describing only the metallic region because of the magnematical investigation of the limit $U / W \rightarrow \infty$. In the light of the more general theory presented here it is rather obvious that it was so (cf. end of part II and part V). Equation 22 does not take into account the entropy term and can be applied only when $T_{\mathrm{L}}$ given by equation 23 is small enough.

[22] Anderson (P. W.), Comments on Solid State Physics, $1970,11,6$.

Edwards (J. M.) and Thouless (D. J.), to be published.

[23] Motт (N. F.), Advances in Physics, 1967, 16, 49.

[24] As we are only interested here in qualitative features of the phase diagram we use the simple free energy of the non magnetic metal. A detailed calculation would however require first to compute the magnetic moment in the metallic state by minimizing its free energy and then to compare it with the free energy of the insulating phase. This approximation make the transition to be a first order one at the critical point where the transition line ends contrary to what one can expect.

[25] Rice (T. M.) and MC Whan (D. B.), I. B. M., J. Res. Develop, 1970, 4, 205.

Cyrot (M.), Advances in Physics, to be published.

[26] Kaplan (T. A.) and Bari (R. A.), J. Appl. Phys., 1970, 41, 875 and Proceedings of the Tenth International Conference on the Physics of Semiconductors, edited by S. P. Keller, J. C. Hensel and F. Stern, US Atomic Energy Commission (1970). Our results disagree with the result of these authors.

[27] MC Whan (D. B.), Rice (T. M.) and RemeiKa (J. P.), Phys. Rev. Letters, 1969, 23, 1384.

Rice (T. M.), Mc Whan (D. B.), I. B. M., J. Res. Develop., 1970, 14, 251.

[28] Motr (N. F.) and Zinamon (Z.), Reports on Progress in Physics, 1970, 33, 881.

[29] Stratonovich (R. L.), Sov. Phys. Dokl., 1958, 2, 416. 